Rev Chil Salud Pública 2012; Vol 16 (3): $247-255$

Estudio de caso
CLAUDIO A. MÉNDEZ(1),

CHRISTIAN MIRANDA J.(2),

M. CRISTINA TORRES(1),

MYRIAM MÁRQUEZ ${ }^{(3)} \mathrm{Y}$

THOMPSON MATTHEWS(4)

(1)Instituto de Salud Pública. Facultad de Medicina. Universidad Austral de Chile. Avenida

Senador Carlos Acharán Arce. Campus Isla Teja. Valdivia. Chile.claudiomendez@uach.cl (2) Departamento de Educación. Facultad de Ciencias Sociales.

Universidad de Chile. Santiago. Chile.

(3)Instituto de Salud Sexual y Reproductiva. Facultad de Medicina. Universidad Austral de Chile. Valdivia. Chile.

(4)Escuela de Química y Farmacia. Facultad de Ciencias. Universidad Austral de Chile. Valdivia. Chile.

Investigación patrocinada por la Dirección de Investigación y Desarrollo de la Universidad Austral de Chile. Código S-2010-37.

\section{Implementación de la política de hospitales autogestionados en Chile: percepción de los profesionales hospitalarios*}

\author{
POLICY IMPLEMENTATION IN INDEPENDENTLY MANAGED HOSPITALS IN CHILE: \\ PERCEPTION OF PROFESSIONAL HOSPITAL STAFF
}

\section{RESUMEN}

Introducción: La reforma de la salud introdujo la figura de los Establecimientos de Autogestión en Red. La investigación tuvo por objetivo explorar las percepciones de los profesionales hospitalarios con relación a la etapa de implementación de la política de Establecimientos de Autogestión en Red en un hospital de alta complejidad en el sur de Chile. Material y método: Estudio cualitativo de caso único holístico basado en entrevistas semi-estructuradas en profundidad a profesionales de la salud del Hospital San José de la ciudad de Osorno durante el período de agosto de 2010 a diciembre de 2011. Se seleccionó una muestra intencionada de 19 profesionales. Se realizaron 19 entrevistas, las cuales fueron grabadas y transcritas en forma literal. El análisis de las entrevistas se realizó mediante la técnica de análisis de contenido en su aproximación convencional. Resultados: Para los entrevistados, la conceptualización de la autogestión está determinada por la autonomía para la toma de decisiones respecto de la asignación de recursos y el financiamiento. Asimismo, las debilidades se relacionaron a la ausencia de capacidades organizacionales y competencias gerenciales de los equipos de salud para la implementación de los cambios. Para los profesionales, para mejorar la etapa de implementación se deben incluir opciones de políticas de recursos humanos y de financiamiento de la función de provisión. Discusión: La política de autogestión hospitalaria es conceptualizada desde la autonomía financiera. Asimismo, la implementación ha estado determinada por las brechas que persisten entre el diseño e implementación de la política de autogestión hospitalaria en red.

Palabras clave: política de salud, servicios de salud, administración

\section{POLICY IMPLEMENTATION IN INDEPENDENTLY MANAGED HOSPITALS IN CHILE: PERCEPTION OF PROFESSIONAL HOSPITAL STAFF}

\section{ABSTRACT}

Introduction: Health reforms have introduced independently managed health care establishments into the health care network. This study explored the perceptions of professional hospital workers with respect

* Resultados preliminares fueron presentados en las XXIX Jornadas Chilenas de Salud Pública 2011. 
to the current stage of policy implementation independently managed establishments, in a high complexity hospital in the south of Chile. Materials and methods: Qualitative single case study based on semi-structured in-depth interviews conducted with health professionals at the San Jose Hospital in the city of Osorno, from August 2010 to December 2011. A sample of 19 professionals was chosen, and the interviews were recorded and transcribed. The analysis was carried out using conventional content analysis. Results: For the interviewees, independent management was conceptualized as autonomy in the decision making process, with respect to resource distribution and financing. Likewise, recognized weaknesses were related to the absence of organizational capacity and competency within management for implementing changes. According to these professionals, policy options for human resources and for financing of health services provision should be included in order to improve the implementation stage. Discussion: Independent hospital management is conceptualized mainly as financial autonomy. Likewise, implementation has been stalled by persisting gaps between design and implementation in independent hospital management policy.

Key words: health policy, health services, hospital administation, health systems.

\section{INTRODUCCIÓN}

Para los sistemas de salud, la relevancia de los hospitales en la provisión de servicios de salud ha implicado que estos se constituyan como un aspecto central en las propuestas de reforma en América Latina. Es así como en las últimas décadas, distintos países han implementado reformas en la organización hospitalaria tendientes a otorgar mayores espacios de autonomía y eficiencia en el ámbito de la gestión ${ }^{1-8}$.

En Chile, previo a la reforma de la salud, los hospitales públicos se caracterizaban por su dependencia de los servicios de salud y no contaban con autonomía para la gestión de sus recursos por parte de los equipos directivos ${ }^{9}$. La entrada en vigencia de la reforma involucró la creación e implementación de los Establecimientos de Autogestión en Red (EAR), los cuales serían aquellos que, dependientes de los servicios de salud, tengan mayor complejidad técnica, desarrollo de especialidades, organización administrativa y número de prestaciones ${ }^{10}$. Para estos hospitales, el cambio implica espacios de mayor flexibilidad y autonomía para la asignación de sus recursos y en la gestión de su presupuesto ${ }^{11}$.

Inicialmente se planteó enero de 2009 como fecha límite para que los 59 hospitales incluidos en la Ley de Autoridad Sanitaria y Gestión se adscribieran al régimen de autogestión, no obstante, al 2008 solo 11 de estos estableci- mientos habían completado el proceso que los acreditaba como EAR ${ }^{12}$. Actualmente, los 59 hospitales descritos en la Ley se encuentran adscritos al régimen de EAR. Sin embargo, no existe evidencia empírica publicada respecto de las dificultades de los equipos en la etapa de implementación de los cambios, así como de los nudos críticos que aún persisten en su implementación.

La presente investigación tiene por objetivo explorar la percepción de los profesionales hospitalarios con relación a los principales cambios organizacionales en la implementación de la política de autogestión hospitalaria en red en un hospital de alta complejidad del sur de Chile.

\section{MATERIAL Y MÉTODO}

Se condujo un estudio de caso cualitativo único holístico. Este tipo de diseño se basa en la selección de una unidad de análisis ${ }^{13}$. En la presente investigación, la unidad de análisis la constituyó la implementación de la política de autogestión en el Hospital San José de la ciudad de Osorno.

Los sujetos informantes correspondieron a un universo de 557 profesionales de la salud que se desempeñaba en los ámbitos clínicos y administrativos del Hospital San José de la ciudad de Osorno. Este centro asistencial es el único hospital de alta complejidad de la red asistencial de la Provincia de Osorno. Se seleccionó una 
249 Implementación de la política de hospitales autogestionados en Chile: percepción de los profesionales hospitalarios - Claudio Méndez et al

muestra por conveniencia ${ }^{14}$ de 19 informantes. Todos ellos profesionales que, al momento de la investigación, presentaban una relación contractual con la institución de al menos dos años. Este criterio buscó cautelar la participación de los profesionales en la etapa de implementación del EAR.

Los profesionales fueron contactados en su institución en forma personal por el equipo de investigadores, previa solicitud de autorización en las instancias administrativas respectivas y aprobación del proyecto por el Comité de Ética del Servicio de Salud de Osorno. Hubo rechazo por parte de 6 profesionales a participar de la investigación, los cuales adujeron causas relacionadas a desconocimiento del tema y disponibilidad de agenda. En la Tabla 1 se describen las características de los sujetos informantes.

El trabajo de campo se realizó entre agosto de 2010 a diciembre de 2011. Para la recolección de la información se utilizó la técnica cualitativa de entrevista individual semi-estructurada en profundidad ${ }^{15}$. Los tópicos del instrumento abordaron los cambios introducidos a la gestión hospitalaria por la reforma de la salud (Tabla 2). Se buscó la saturación global y condensación de las dimensiones previamente descritas, lo cual determinó el tamaño muestral final. Las entrevistas tuvieron una duración mínima de 20 minutos y máxima de 1 hora y se realizaron en las dependencias de la institución laboral de los informantes. Las entrevistas fueron conducidas por uno de los autores, con el fin de obtener consistencia y reducir la variación de la aproximación a los temas presentados a los entrevistados $^{16}$. Los discursos fueron grabados, previo conocimiento por parte del entrevistado y suscripción del respectivo documento de consentimiento informado, y transcritas en forma literal. Posteriormente -como criterio de calidad- fueron enviadas a los entrevistados para obtener comentarios y correcciones.

Las entrevistas transcritas fueron leídas en forma reiterada por el grupo de investigadores y codificadas de acuerdo a la técnica de análisis del contenido en su aproximación inductiva o convencional ${ }^{17,18}$. Para la reducción de la información, se definieron esquemas de codificación (Tabla 3), que posteriormente permitieron determinar las categorías y subcategorías temáticas.
Tabla 1. Características de la muestra de los profesionales entrevistados del Hospital San José de Osorno.

\begin{tabular}{|c|c|c|}
\hline Variable & $\mathrm{N}$ & $\%$ \\
\hline \multicolumn{3}{|l|}{ Sexo } \\
\hline Masculino & 7 & 37 \\
\hline Femenino & 12 & 63 \\
\hline Total & 19 & 100 \\
\hline \multicolumn{3}{|l|}{ Edad } \\
\hline $20-30$ & 1 & 5 \\
\hline $31-40$ & 4 & 21 \\
\hline $41-50$ & 4 & 21 \\
\hline $51-60$ & 9 & 47 \\
\hline $61-70$ & 1 & 5 \\
\hline Total & 19 & 100 \\
\hline \multicolumn{3}{|l|}{ Profesión } \\
\hline Médico cirujano & 8 & 42 \\
\hline Enfermera & 2 & 11 \\
\hline Tecnólogo médico & 1 & 5 \\
\hline Asistente social & 1 & 5 \\
\hline Kinesiólogo & 1 & 5 \\
\hline Nutricionista & 1 & 5 \\
\hline Matrona & 1 & 5 \\
\hline Químico farmacéutico & 3 & 16 \\
\hline Abogado & 1 & 5 \\
\hline Total & 19 & 100 \\
\hline
\end{tabular}

\section{Años de Servicio}

\begin{tabular}{lrr}
$01-10$ & 9 & 47 \\
$11-20$ & 1 & 5 \\
$21-30$ & 4 & 21 \\
$31-40$ & 5 & 26 \\
Total & 19 & 100 \\
\hline
\end{tabular}

Seguido, se seleccionaron citas literales de las entrevistas, de acuerdo al criterio de recurrencia de los conceptos e ideas que emergieron de las 
Tabla 2. Guía de entrevista semi-estructurada en profundidad.

\begin{tabular}{cl}
\hline Guion de Preguntas \\
\hline - ¿Qué entiende usted por autogestión? \\
- ¿Cuáles cree usted son los propósitos de autogestionar los hospitales? \\
- ¿Cuáles cree usted son las consecuencias para la gestión hospitalaria? \\
- ¿Cuáles han sido los obstáculos organizacionales para implementar la autogestión? \\
- ¿Cuáles han sido las fortalezas organizacionales para la implementación? \\
- ¿Cuáles han sido las fortalezas de los equipos para la implementación? \\
- ¿Qué cambios cree usted son necesarios para mejorar la autogestión? \\
\hline
\end{tabular}

entrevistas $^{19}$. Se utilizaron códigos para las citas de los informantes, con el fin de cautelar su anonimato.

La calidad de la investigación se abordó a través de los criterios de rigor científico de credibilidad, dependencia, confirmabilidad y transferibilidad ${ }^{20-22}$. La credibilidad a través de la triangulación de investigadores y el envío de las transcripciones a los entrevistados. La dependencia, confirmabilidad y transferibilidad mediante la descripción sistemática de la aproximación metodológica utilizada y reflexividad en el análisis.

\section{RESULTADOS}

En la Tabla 4 se presentan las categorías y subcategorías temáticas que emergieron del análisis de las entrevistas. Los resultados se describen de acuerdo a cada categoría temática y sus respectivas subcategorías.

\section{Autogestión en red}

Para los entrevistados, la conceptualización de la autogestión en red está determinada por la percepción de autonomía en la gestión de las instituciones hospitalarias. Principalmente, se identifica la autonomía en el ámbito de la financiación como el elemento central de la conceptualización. En el mismo sentido, sustentan dicha precepción en la autonomía para la toma de decisiones respecto de la asignación de recursos financieros a través de la implementación de los centros de costo.

[01-HSJ] “...Significa que el hospital debe ser capaz de sustentarse económicamente, porque antiguamente recibíamos todo de afuera y ahora tenemos por medio de todos los centros de costos una cantidad de dinero necesaria para poder llevar adelante el hospital..."

Por otra parte, la construcción de autogestión hospitalaria por parte de los profesionales, involucra la autonomía y descentralización en la toma de decisiones dirigidas a mejorar la provisión de servicios de salud.

[04-HSJ] “...Para mí, es poder decidir en forma local decisiones que se acerquen más a las necesidades de las personas que estoy destinada a atender y que tienes que hacer llegar los recursos necesarios con el recurso humano que se requiere para dar una atención, tener definida la cartera de servicios que tienes de disponibilidad para la población objetivo y poder administrar todo lo que está en ese sentido para dar mejores resultados..."

Desde el ámbito del diseño de la autogestión hospitalaria, para los entrevistados, los elementos que determinaron su diseño obedecieron a la necesidad de introducir estrategias dirigidas a cautelar por una ejecución presupuestaria eficiente por parte de las instituciones hospitalarias.

[08-HSJ] “...Bueno, yo siempre he dicho que la salud es un saco sin fondo, eso como primera cosa. Y lo otro es ordenar, ordenar todos los establecimientos y ver en qué se está gastando el dinero que ingresa de las arcas fiscales, ver cómo se están gestionando, las digamos, todas las metas que tienen los hospitales..."

En el mismo sentido, para los entrevistados, el diseño de la política de autogestión hospitalaria en red privilegió la necesidad de mejorar la eficiencia en la ejecución presupuestaria de 
los hospitales, junto con una optimización en la gestión de las instituciones.

[07-HSJ] "...Yo pienso que va a llegar a ser bueno porque es como que estamos obligados a trabajar más, generar nuestros propios recursos y no estar esperando sentaditos que a fin de mes lleguen, por decir algo, mil millones para cubrir las necesidades..."

Para los profesionales, la conceptualización y objetivos del diseño de la autogestión hospitalaria se perciben desde la autonomía para la asignación de recursos financieros.

\section{IMPLEMENTACIÓN}

La implementación de la política de autogestión hospitalaria implicó la adaptación de los equipos de salud a nuevas instancias organizacionales y administrativas, determinada por la asimetría de información entre los profesionales con responsabilidades directivas de aquellos que se desempeñan solo en el ámbito clínico.

Sin embargo, ha sido la implementación de los centros de responsabilidad y de costos, la asimetría de información, falta de sistemas de registros y la ausencia de competencias gerenciales las que, para los entrevistados, se han constituido como las principales dificultades en la etapa de implementación.

[13-HSJ]"...Existe una estructura dentro del hospital que considera un funcionamiento dado por un precepto legal que hoy día está derogado. Entonces se aplicaron conceptos que están en la nueva Ley de Autoridad Sanitaria como centro de responsabilidad y centro de costo. No obstante, reconociendo que esos conceptos son buenos, este tránsito que ha tenido que suceder desde el decreto 42 -que es el que organizaba la estructura hospitalaria del servicio- a la nueva estructura, no ha cuajado bien, no ha habido un engranaje como corresponde. O sea estábamos estructurados de una cierta manera, cierto. Hoy día lo que hicimos con esa misma estructura fue darles nombres como centros de responsabilidad..."

Asimismo, otros aspectos claves para los equipos en la implementación de la autogestión hospitalaria se han concentrado en las brechas

de información y coordinación asistencial para la implementación de los cambios, lo que ha implicado incertidumbre en los equipos respecto del impacto de la autogestión para el desempeño de las instituciones.

[18-HSJ] "...Como dificultad para esto es el tema de temor que sentimos los más nuevos y de cargos no directivos que si se quedan cortos de plata; nos den el sobre azul. Lo otro, que es la gente de los escalafones de servicios no tienen idea de quétrata todo esto y están convencidos, al igual que algunos colegas, que se trata del inicio de un proceso de privatización..."

Por otra parte, para los profesionales, la necesidad de políticas y estrategias en el área de recursos humanos en salud se ha evidenciado en las dificultades para la implementación del modelo de gestión en las instituciones hospitalarias.

[19-HSJ] “...Principalmente la falta de recurso humano competente, la falta de educación al personal sobre el tema, la falta de sistemas informáticos actualiza-

Tabla 3. Unidades de análisis y esquema de codificación.

Unidad de Análisis

Autogestión

Recursos humanos

Políticas

Provisión

\section{Código}

Concepto
Objetivo
Diseño
Implementación
Evaluación

Garantías

Gestión

Modelo

Integración

Regulación
Distribución
Retención
Desempeño
Competencias
Regulación

Financiamiento

Recursos humanos

Provisión

Regulación

Integración 
dos para gestión administrativa y contable. En general, a mi parecer, el hecho de que nuestro hospital haya sido incorporado a los EAR por fuerza de ley es un error garrafal, te preguntarás por qué, y es simple, porque no se puede autogestionar cuando las condiciones básicas no existen..."

Como parte de la necesidad de políticas y estrategias, la ausencia de capacitación en nuevas competencias profesionales alineadas con la política de autogestión ha sido el principal obstáculo para la implementación de la autogestión hospitalaria.

[06-HSJ] “...Para mí la mayor dificultad ha sido el tema de la capacitación. Por ejemplo, yo jamás he recibido capacitación formal de la institución, todo lo que yo sé es porque hice un diplomado el año pasado, pagado por otra vía, yo me lo pagué..."

La implementación de los cambios que implica el régimen de autogestión, ha sido determinada por la débil coordinación asistencial, así como brechas del diseño que ha trascendido a la etapa de implementación.

\section{Opciones de políticas}

Para los entrevistados, aspectos del financiamiento, asignación de recursos y de la política y gestión de recursos humanos se constituyen como los principales ámbitos para el diseño e implementación de políticas y estrategias que permitan mejorar el desempeño de las instituciones hospitalarias bajo el régimen de autogestión.

En el ámbito de los recursos humanos en salud, abordan la necesidad de una política de

Tabla 4. Categorías y subcategorías temáticas.

\begin{tabular}{ll}
\hline Categoría & Subcategoría \\
\hline $\begin{array}{l}\text { Autogestión en } \\
\text { Red }\end{array}$ & $\begin{array}{l}\text { Significado } \\
\text { Objetivos } \\
\text { Diseño } \\
\text { Impacto }\end{array}$ \\
& $\begin{array}{l}\text { Cambios organizacionales } \\
\text { Omplementación } \\
\text { Fortáculos organizacionales } \\
\text { Fortalezas organizacionales } \\
\text { Equipos de salud }\end{array}$ \\
políticas & $\begin{array}{l}\text { Financiamiento } \\
\text { Recursos humanos }\end{array}$ \\
\hline
\end{tabular}

recursos humanos desde la capacitación y entrenamiento gerencial dirigido al desarrollo de competencias alineadas a los nuevos procesos clínicos y administrativos.

[02-HSJ] “...Lo primordial es generar un cambio de mentalidad en el recurso humano, para lograr alinear a todos tras un mismo objetivo. Se deben mejorar todos los procesos y capacitar a los funcionarios en este nuevo modelo de gestión..."

Por otra parte, para los informantes, mejorar el desempeño de los equipos de salud pasa por determinar un mejor sistema de designación de profesionales que ejerzan responsabilidades directivas en las instituciones de provisión de servicios de salud. Asimismo, avanzar en más y mejores sistemas de medición del desempeño de los equipos.

[12-HSJ] “...Lamentablemente al buen elemento, a la persona que es eficiente, la persona que cumple a cabalidad con sus funciones lo premias dándole más pega, y a los flojos, a los que, -que los hay como en todos lados, como en todas partes-, no les piden nada, no, este no lo va a hacer, para qué vas a perder tu tiempo en pedirle esa pega si no lo va a hacer. Entonces claro, entonces los buenos, los pobres ya no dan más y los flojos cada día más contentos. Entonces, ese sistema como que nos juega en contra esa parte $y$, además, es por el problema de las calificaciones, porque las calificaciones son un chiste, todos somos excelentes, todos tenemos puros sietes..."

En el ámbito del financiamiento, los entrevistados identifican como prioritario mejorar el sistema de asignación de recursos. Específicamente un sistema que permita la descentralización de los presupuestos para las instituciones.

[11-HSJ] “...Bueno, yo creo desde el punto de vista macro como siempre, mejorar la infraestructura y los recursos de dinero que deben fluir hacía los hospitales directamente y no a través de los servicios..."

Asimismo, identifican el avanzar en mejores sistemas de diagnóstico y monitoreo de los resultados clínicos y administrativos, así como mejorar los sistemas de selección de directivos. 
[05-HSJ] “...Hacer un buen proceso de diagnóstico, ver dónde nos falta, clínicas o administrativas, y después supervisar, armar pautas de monitoreo para poder ver los resultados, porque si no monitoreas, cualquier decisión que tomes puede estar equivocada, porque para tomar decisiones necesitas tener algo que te dé respaldo..."

Las opciones de políticas identificadas por los profesionales, difieren entre aquellas que buscan un cambio estructural respecto de las que se plantean en el ámbito administrativo de la propia institución hospitalaria.

\section{DISCUSIÓN}

Los hallazgos obtenidos en la investigación permiten identificar las principales brechas que los profesionales de la salud del Hospital San José de la ciudad de Osorno perciben respecto del diseño e implementación de los hospitales de autogestión en red. Asimismo, identifican las principales dificultades que los equipos han enfrentado para la implementación de los cambios.

Para los profesionales, la percepción respecto de lo que implica la autogestión hospitalaria está determinada por la autonomía respecto de la toma de decisiones, la asignación de recursos y gestión financiera. Si bien la autogestión hospitalaria en Chile no está relacionada a una completa autonomía de los hospitales respecto de la función de financiación del sistema, la percepción de los profesionales se alinea a los objetivos de la autonomía hospitalaria como una política basada en la contención de costos y la eficiencia hospitalaria ${ }^{23,24}$. No obstante, un resultado relevante respecto de dicha concepción se relacionó a la ausencia del concepto de redes asistenciales como parte de la autogestión hospitalaria.

Por otra parte, la etapa de implementación de otras experiencias de autonomía hospitalaria en América Latina ha presentado como factor común, y obstáculo para la implementación, el alto grado de incertidumbre organizacional en los equipos de salud ${ }^{25,26}$. En el caso de la implementación de la autogestión hospitalaria en el hospital estudiado, la incertidumbre ha estado relacionada principalmente a los niveles de coordinación dentro y entre las propias organizaciones hospitalarias, así como de los lineamien- tos de parte de la rectoría del sistema.

Otro hallazgo se relacionó con la percepción respecto de la rotación de directivos hospitalarios en la etapa de implementación. No obstante dicha situación fue destacada como una de las mayores dificultades de los establecimientos para acceder a la categoría de EAR previa modificación del plazo legal ${ }^{27}$, para los profesionales continúa representando un obstáculo y brecha entre el diseño e implementación. Esta situación puede explicarse por la dificultad de retener a directivas(os) a través de proyectos e incentivos atractivos en las instituciones hospitalarias. Al respecto, otras investigaciones han planteado la falta de reconocimiento y de compromiso más que aspectos pecuniarios, como los principales factores desmotivacionales en los directivos hospitalarios en Chile ${ }^{28}$.

Asimismo, si bien la ausencia de una política de recursos humanos para acompañar los cambios en las organizaciones hospitalarias ha sido advertida como una brecha determinante entre el diseño e implementación ${ }^{29-32}$, a siete años de la aprobación de los cuerpos legales de la reforma esta continúa siendo uno de los factores determinantes para la implementación y el desempeño de los equipos e instituciones. Más aún, la ausencia de políticas y estrategias en recursos humanos en salud fundamenta que esta área sea una de las opciones de políticas identificadas por los entrevistados como determinantes para mejorar la implementación.

La ausencia de competencias organizacionales y estrategias de capacitación para los equipos de salud ha impedido alcanzar los estándares que la autogestión demanda para la institución. Existe evidencia que señala la importancia de la evaluación de los procesos de gestión con objetivo de mejorar la satisfacción de los usuarios en los hospitales públicos en Chile $^{33}$. En el caso de la autogestión, avanzar en la inclusión de los recursos humanos como factor determinante de los procesos de gestión será fundamental para entregar una mejor provisión de servicios de salud y percepción de satisfacción usuaria.

Finalmente, si bien los resultados de la investigación se limitan al análisis de los discursos de profesionales pertenecientes a un caso analizado, la homogeneidad estructural de la 
función de provisión pública del sistema de salud chileno permite identificar aspectos claves que podrían ser considerados para la etapa de implementación en la red hospitalaria de alta complejidad del país con similares características al caso estudiado. Por otra parte, los resultados obtenidos plantean la necesidad de conducir nuevas investigaciones dirigidas a medir el impacto de la implementación de la autogestión hospitalaria en el desempeño de los equipos de salud y de los hospitales.

\section{Referencias}

1. hernández M. Reforma sanitaria, equidad y derecho a la salud en Colombia. Cad Saúde Pública 2002; 18(4): 991-1001.

2. Echeverri O. Mercantilización de los servicios de salud para el desarrollo: el caso de Colombia. Rev Panam Salud Pública 2008; 24(3): 210-216.

3. Homedes N, Ugalde A. Las reformas de salud neoliberales en América Latina: una visión crítica a través de dos estudios de caso. Rev Panam Salud Publica 2005; 17(3): 210-220.

4. LLoyd-Sherlock P. Health sector reform in Argentina: a cautionary tale. Social Science \& Medicine 2005; 60: 1893-1903.

5. Belmartino $\mathrm{S}$. Una década de reforma de la atención médica en Argentina. Salud Colectiva 2005; 1(2): 155171.

6. Jack W. Contracting for health services: an evaluation of recent reforms in Nicaragua. Health Policy Plan 2003; 18(2): 195-204.

7. Garcia-Prado A, Chawla M. The impact of hospital management reforms on absenteeism in Costa Rica. Health Policy Plan 2006; 21(2): 91-100.

8. Mesa-Lago C. Social security in Latin America: pension and health care reforms in the last quarter century. Latin American Research Review 2007; 42(2): 181-201.

9. Nancuante U, Romero A. La reforma de la Salud. 1a $^{\mathrm{a}}$ ed. Santiago de Chile: Editorial Biblioteca Americana, 2008.

10. Ley 19.937 de Autoridad Sanitaria y Gestión. Publicada en el Diario Oficinal el 24 de Febrero de 2004, Chile.

11. Retamal M. Autogestión hospitalaria en cifras. Rev Chil Salud Pública 2009; 13(3): 169-174.

12. Artaza O. Los desafíos de la autogestión hospitalaria. Rev Chil Pediatr 2008; 79: 127-30.

13. Yin RK. Case study research: design and methods. Sage $4^{a} \mathrm{Ed}$. 2009. Estados Unidos. 219 páginas.
14. Onwuegbuzie AJ, Leech NL. A call for qualitative power analyses. Quality \& Quantity 2007; 41: 105121.

15. Dicicco-Bloom B, Crabtree BF. The qualitative research interview. Medical Education 2006; 40: 314321.

16. Fitzpatrick R, Boulton M. Qualitative methods for assessing health care. Qual. Saf. Health Care 1994; 3: 107-113.

17. Hsieh H, Shannon SE. Three approaches to qualitative content analysis. Qual Health Res 2005; 15(9): 1277-1288.

18. Elo $S$, Kyngäs $H$. The qualitative content analysis process. Journal of Advanced Nursing 2008; 62(1): 107-115.

19. Ulin P, Robinson E, Tolley E. Qualitative Methods in Public Health: A field guide for applied research. Jossey-Bass $1^{a}$ Ed. 2005. Estados Unidos. 318 páginas.

20. Onwegbuzie AJ, Leech NL. Validity and qualitative research: an oxymoron? Quality \& Quantity 2007; 41: 233-249.

21. Tobin GA, Begley CM. Methodological rigour within a qualitative framework. Journal of Advanced Nursing 2004; 48(4): 388-396.

22. Malterud K. Qualitative research: standards, challenges, and guidelines. Lancet 2001; 358: 483-88.

23. Unger JP, De Paepe P, Ghilbert P, Soors W, Green A. Disintegrated care: the Achilles heel of international health policies in low and middle-income countries. International Journal of Integrated Care 2006; 6(18): 1-13.

24. Sharma S, Hotchkiss D. Developing financial autonomy in public hospitals in India: Rajasthan's model. Health Policy 2001; 55: 1-18.

25. Mcpake B, Yepes F, Lake S, Sanchez L. Is the Colombian health system reform improving the performance of public hospitals in Bogotá? Health Policy Plan 2003; 18(2): 182-94.

26. Machado AL, Giacone M, Alvarez C, Carri P. Health reform and its impact on healthcare workers: a case study of the National Clinical Hospital of Cordoba, Argentina. Social Medicine 2007; 2(4): 156-164.

27. Sociedad Chilena de Administradores en Atención Médica y Hospitalaria. Los desafíos pendientes de la autogestión hospitalaria en red. Cuad Med Soc (Chile) 2009; 49: 171-77.

28. Bustamante-Ubilla MA, del Río-Rivero MC, LobosAndrade GE, Villarreal-Navarrete PI. Percepción de la motivación de los directivos intermedios en tres hospitales de la Región del Maule, Chile. Salud Publica Mex 2009; 51: 417-426.

29. Méndez CA. Los recursos humanos de salud en Chile: el desafío pendiente de la reforma. Rev Panam Salud Pública 2009; 26(3): 276-80. 
30. Méndez CA. Reflexión sobre la planificación de los recursos humanos y la autonomía de gestión en los hospitales de Chile. Rev Esp Salud Pública 2009; 83: 371-378.

31. Méndez CA, Torres MC. Autonomía en la gestión hospitalaria en Chile: los desafíos para el recurso humano en salud. Rev. Saúde Pública 2010; 44(2): 366-371.

32. Méndez CA, Alarcón A. Alineación del recurso hu- mano y regulación de la competencia como factores claves para la autogestión hospitalaria en Chile. Rev Chil Salud Pública 2011; 15(2): 90-97.

33. Riveros $\mathrm{J}$, Berné $\mathrm{C}$. Análisis de la opinión de usuarios sobre calidad percibida y satisfacción con hospitales públicos: estudio de caso desde la perspectiva de la aplicación del marketing. Rev Méd Chile 2007; 135 : 862-870. 